\title{
Research of Precise Measurement Technology of Automatic Production Line
}

\author{
Zhihong Wang ${ }^{1}$, Wenbin Wang ${ }^{2}$, Yuqin Yao ${ }^{1, a}$ \\ ${ }^{1}$ Chengdu University of Information Technology, Chengdu, 610225, China \\ ${ }^{2}$ Yalong River Hydropower Development Company LTD, Chengdu, 610051, China \\ ${ }^{a}$ email: yyq@cuit.edu.cn
}

Keywords: Camera calibration; Nonlinear optimization; Digital image processing; Edge detection

\begin{abstract}
Digital image processing is to modify graphics, improving image quality and extract valuable information from the images. The detection technology based on machine vision is more applicable for the dangerous environment and situations that difficult to find, it is can also avoid the error generated by human vision. This paper introduces the precise measurement technology based on machine vision, and distortion correction theory was studied by the nonlinear optimization which is based on the traditional camera calibration method, then the samples were measured by edge detection with Sobel operator. The result shows that this method is promotive to the accurate measurement.
\end{abstract}

\section{Introduction}

Machine vision can replace human visual by imaging tool, by which the structure of object in both 2D and 3D will be extracted from the original image and the objcet's properties was described. In machine vision measurement technology, camera calibration is the core research content. The optical parameter and internal parameters can be calculated by the corresponding model which is established based on the known object, if we get these parameters, the camera distortion can be carried out $^{[1]}$. It is known that image enhancement plays a key role in image processing which is the basis of machine vision measurement, meanwhile the edge of image contains a large number of important information, they are both the key parameters in camera calibration which is vital to the accurate measurement in Automated production line.

\section{Camera calibration}

\section{(1)The calibration process}

The camera calibration is the process that calculating the internal and external parameters of the camera. In this paper, the checkerboard plane target is chosen which is easy to operate and precisely to accurate ${ }^{[2]}$. As we determine the projection relationship between the targets and image, the camera calibrate can on its way. In the world coordinate system, the calibration tag can be expressed as $M_{i}$. Calibration plate is placed in the world coordinate system, we get the conclusion that $\mathrm{Z}=0$, because that the calibration plate is flat ${ }^{[3]}$. The coordinates of the center point is marked as $m_{i}$ in the image, Coordinate $\pi\left(M_{i}, c\right)$ is obtained by projection transformation, we can calculate the camera parameters by expression (1).

$$
d(c)=\sum_{i=1}^{l} \sum_{i=1}^{k}\left\|m_{i, j}-\pi\left(M_{i}, c\right)\right\|^{2} \rightarrow \min
$$

In expression (1), the Coordinate $m_{i, j}$ is stand for point $i$ in image $j$. To make a higher accuracy camera parameters, the four corners of image should be covered with calibration board. The accuracy of the distortion coefficient is low in the case of unconsidering the distortion of the corner. 
For example, an image was optimized, in which its center coordinate $m_{i}$ and projection coordinate $\pi\left(M_{i}, c\right)$ can be respectively expressed as $\left(U_{i}, V_{i}\right)$ and $\left(u_{i}, v_{i}\right)$. The target expression can be optimized by expression (2). For optimal performance, $F(x)$ must be minimized, and the error of the projection can be minimized by Least Square Method (LSM) to:

$$
\begin{array}{r}
F(x)=\sum_{i=1}^{m} f_{i}^{2}(x) \\
f_{i}^{2}(x)=\left(U_{i}-u_{i}\right)^{2}+\left(V_{i}-v_{i}\right)^{2} .
\end{array}
$$

Supposing that $f_{i}(x)$ is a nonlinear function and $F(x)$ have continuous partial derivatives. The nonlinear least squares can be obtained by linear least squares. Assume $f_{i}(x)$ as expression (4):

$$
f_{i}(x)=p_{i}^{T} x-b_{i}
$$

In expression (4), $p_{i}$ is a n-dimensional column vector and $b_{i}$ is the real number. And we can express these in terms of matrix multiplication, as follows.

$$
\begin{gathered}
A=\left[\begin{array}{c}
p_{1}^{T} \\
p_{2}^{T} \\
\bullet \\
\bullet \\
p_{m}^{T}
\end{array}\right] \quad \boldsymbol{b}=\left[\begin{array}{c}
b_{1} \\
b_{2} \\
\bullet \\
\bullet \\
b_{m}
\end{array}\right] \\
F(x)=\sum_{i=1}^{m} f_{i}^{2}(x)=\left(f_{1}(x), f_{2}(x), \ldots, f_{m}(x)\right)\left[\begin{array}{c}
f_{1}(x) \\
f_{2}(x) \\
\bullet \\
\bullet \\
f_{m}(x)
\end{array}\right]=(A x-b)^{T}(A x-b)=x^{T} A^{T} A x-2 b^{T} A x+b^{T} b
\end{gathered}
$$

The stable point for $F(x)$ can be calculated by expression (5).

$$
\nabla F(x)=2 A^{T} A x-2 A^{T} b=0
$$

As a result, we have the following conclusion by expression (6).

$$
A^{T} A x=A^{T} b
$$

It is usually required that the design matrix A is column full rank in working out the stationary point, we can get the following result.

$$
\bar{x}=\left(A^{T} A\right)^{-1} A^{T} b
$$

\section{(2)Calibration results}

According to the principle of the above, this thesis calibrates the video cameras and obtains their internal parameters.

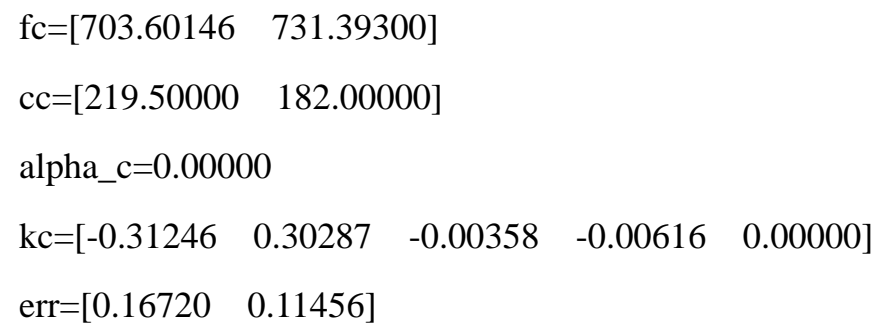

\section{Detection technology}

\section{(1) Image enhancement}

In the process of generation and transmission, the image noise is distinct and they deteriorate image quality. Noise has serious effects on quality of successive image processing ${ }^{[4]}$.The purpose of image enhancement is to improve the visual effect of image. 
Median filter is a kind of local average smoothing technique. In given conditions, it can overcome the loss of image details. The simulation experiment shows that this algorithm can protect image signal detail as much as possible when effectively image noise.

\section{(2) Edge detection}

Edge detection is the important content in the image processing, and it is the basis of image segmentation, pattern recognition and computer version ${ }^{[5]}$. In this paper, we summarize the methods of edge detection and make a contrast among these classical edge detection operators. The results of experiment indicate that it is relatively effective for sobel operator to realize digital image fringe detecting and picking up.

\section{(3)Simulation results}

The research is mainly to measure a book which is $965 \mathrm{~mm} \times 787 \mathrm{~mm}$, the results of the simulation in the example were given, as follows.

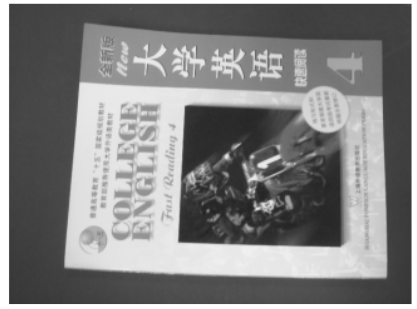

Figure 3.1 the original image

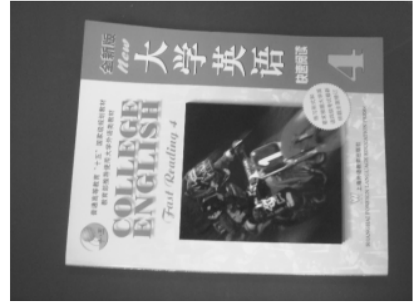

Figure3.2 the image of median filtering

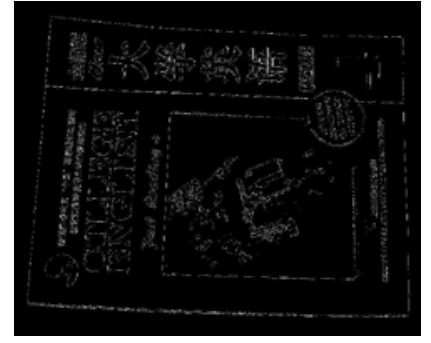

Figure 3.3 the image of edge detection

Then the experimental results of correction are shown below.

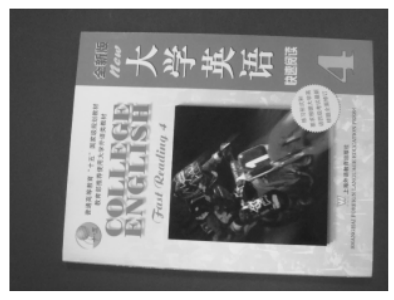

Figure 3.4 the image of distortion correction

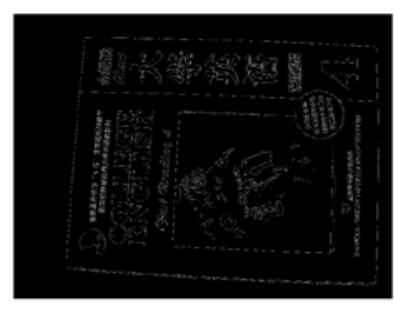

Figure 3.5 the image of edge detection after distortion correction

\section{(4) Measurement results}

In this paper, five images of the book were measured .The results appear as shown below.

Table 1 length measurement

\begin{tabular}{|c|c|c|c|c|c|}
\hline Image sequence number & 1 & 2 & 3 & 4 & 5 \\
\hline The original image (pixel) & 2418 & 2501 & 2327 & 2544 & 2652 \\
\hline The corrected image (pixel) & 2310 & 2403 & 2328 & 2540 & 2649 \\
\hline Size (mm) & 1010.1 & 1004.4 & 964.6 & 966.5 & 966 \\
\hline
\end{tabular}

The average value:

$$
\bar{M}_{1}=\frac{1010.1+1004.4+964.6+966.5+966}{5}=982.3
$$

The average error:

$$
\delta_{1}=\left|\frac{982.3-965}{965}\right| \times 100 \%=1.8 \%
$$


Table 2 width measurement

\begin{tabular}{|c|c|c|c|c|c|}
\hline Image sequence number & 1 & 2 & 3 & 4 & 5 \\
\hline The original image (pixel) & 1225 & 1237 & 1178 & 1394 & 1226 \\
\hline The corrected image (pixel) & 1209 & 1231 & 1253 & 1278 & 1223 \\
\hline Size (mm) & 797.4 & 790.8 & 739.9 & 858.4 & 788.9 \\
\hline
\end{tabular}

The average value:

$$
\bar{M}_{2}=\frac{797.4+790.8+739.9+858.4+788.9}{5}=795.1
$$

The average error:

$$
\delta_{2}=\left|\frac{795.1-787}{787}\right| \times 100 \%=1.1 \%
$$

In comparison with the conventional method, this one proved to 5 books were measured, and the results showed hat the degree of accuracy was high and deviations were minimal.

\section{References}

[1] F. DIERKS. Sensitivity and Image Quality of Digital Cameras. Technical report, Basler AG, October 2004.

[2] AIA. GigE Vision: Camera Interface Standard for Machine Vision, Version 1.0, May 2006.

[3] H. HIRSCHMULLER, P. R. INNOCENT, J. GARIBALDT. Real-time correlation-based stereo vision with reduced border errors. International Journal of Computer Vision, 47(1-3):229-246, 2002.

[4] ISO 15739: 2003. Photography-Electronic Still-Picture Imaging-Noise Measurements, 2003.

[5] C. STEGER. System and method for object recognition. US Patent, 7062 093, June 2006. 